\title{
When light explores space and time
}

\author{
Olivier J. F. Martin \\ Swiss Federal Institute of Technology Lausanne (EPFL), EPFL-STI-NAM, Nanophotonics and Metrology Laboratory, Lausanne, \\ Switzerland
}

We are all familiar with crystal structures built from the periodic arrangement in space of atoms, molecules or even scatterers. Their periodicity is at the origin of a wealth of optical phenomena ranging from birefringence-first observed by Christiaan Huygens in the $17^{\text {th }}$ century ${ }^{1}$ - to optical bandgaps in photonic crystals and their promise to slow down light in the $21^{\text {st }}$ century. ${ }^{2}$ This modulation of the dielectric function is not limited to one, two or three space dimensions, but can also be forced upon the fourth dimension: time! Actually, as early as the beginning of the 1960 s, the idea of modulating the dielectric function in time emerged, ${ }^{3}$ and was later elaborated to control parametric nonlinear processes by virtue of travelling waves, ${ }^{4}$ paving the way for extremely efficient devices, such as traveling-wave photodetectors. ${ }^{5}$ Exploring periodic variations of the dielectric function in all four dimensions of space and time unleashes fascinating physics and provides opportunities for exciting experiments.

In this issue of Advanced Photonics, Zoé-Lise Deck-Léger et al. ${ }^{6}$ offer an extremely insightful analysis of such spacetime crystals, where the dielectric function is modulated periodically in space and time. Exploring all four dimensions of our environment is certainly vertiginous and quite confusing at best. The authors overcome this challenge by focusing on 2D spacetime crystals with periodicity in time and in one space dimension. This way, they are able to use concepts from special relativity and provide explicit pictorial representations of the different optical phenomena associated with simultaneous spatial and temporal periodicities.

Planar interfaces between regions of time/space with different dielectric functions play a key role in the chosen geometry and are treated in detail, leading to a generalization of the Stokes principle for a spacetime interface. Here, this principle is elegantly derived from sole considerations on symmetries supported by the system.

The light line reigns over a spacetime crystal, as it divides it between subliminal and superluminal structures, which are related via spacetime inversion and build two dual electromagnetic problem spaces. These two problem spaces support extremely different solutions for a problem as simple as light transmission through a slab. While the subluminal slab behaves in a way similar to the time-invariant antireflection coating we find on spectacles, with light bouncing forth and back ad infinitum; for the superluminal slab such multiple reflections do not exist and light is merely "transmitted" through the slab at a later moment in time.

With the current pace of technological developments, we can trust that the concepts elaborated by Deck-Léger and colleagues will soon prompt experimental implementations of this new fascinating chapter of photonics, as was the case for the seminal work of Cassedy and Oliner in the 1960s.

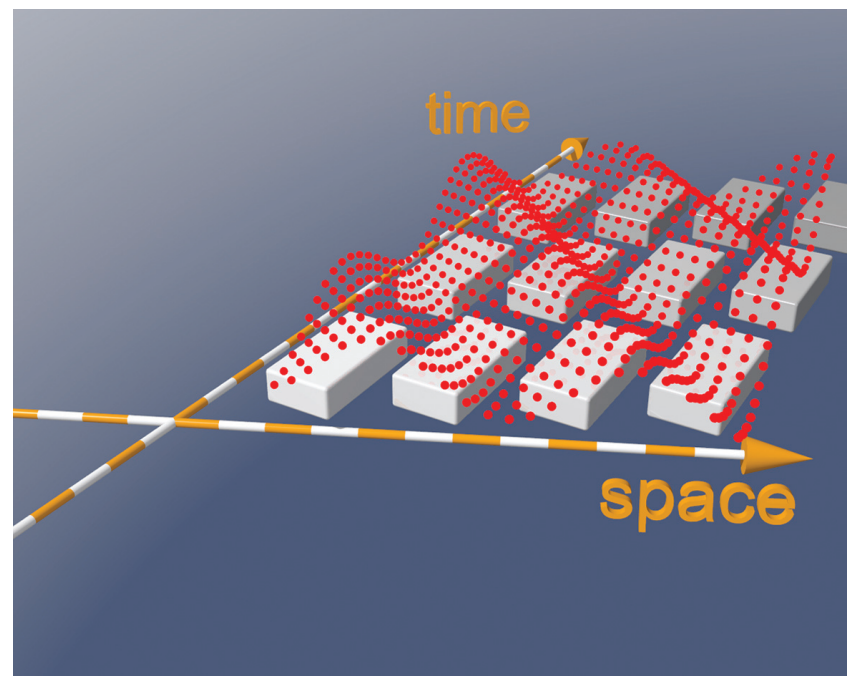

Artistic representation of light interacting with a periodic timespace crystal.

\section{References}

1. J. Z. Buchwald, "Huygens' methods for determining optical parameters in birefringence," Arch. Hist. Exact Sci. 61(1), 67-81 (2007).

2. T. F. Krauss, "Why do we need slow light?" Nat. Photonics 2(8), 448-450 (2008).

3. E. S. Cassedy and A. A. Oliner, "Dispersion relations in time-space periodic media: part I-stable interactions," Proc. IEEE 51(10), 1342-1359 (1963).

4. D. Holberg and K. Kunz, "Parametric properties of fields in a slab of time-varying permittivity," IEEE Trans. Antennas Propag. 14(2), 183-194 (1966)

5. V. M. Hietala et al., "Traveling-wave photodetectors for high-power, large-bandwidth applications," IEEE Trans. Microwave Theory Tech. 43(9), 2291-2298 (1995).

6. Z.-L. Deck-Léger et al., "Uniform-velocity spacetime crystals," Adv. Photon. 1(5), 056002 (2019).

Olivier J. F. Martin is professor of nanophotonics and optical signal processing at the Swiss Federal Institute of Technology Lausanne (EPFL), where he conducts a comprehensive research on the optical properties of plasmonic nanostructures, with applications in trapping, sensing, metasurfaces and nonlinear optics. 